\title{
ASPIRIN RESISTANCE IN NEUROVASCULAR DISEASES
}

\author{
Martin Vališ ${ }^{1}$, Dagmar Krajíčková ${ }^{1}$,Jaroslav Malý2, Radovan Malý2, Ilona Fátorová2, \\ Oldřich Vyšata ${ }^{1,3}$, Roman Herzig ${ }^{1}$
}

University Hospital Hradec Králové and Charles University in Prague, Faculty of Medicine in Hradec Králové, Czech Republic: Comprehensive Department of Neurology, Stroke Center ${ }^{1}$, Department of Internal Medicine ${ }^{2}$; Department of Computing and Control Engineering, Institute of Chemical Technology, Prague, Czech Republic ${ }^{3}$

Summary: Introduction: The issue of resistance to antiplatelet therapy has raised many questions in the area of neurovascular diseases. The first objective of this work was to determine the prevalence of aspirin resistance in neurovascular patients with clinical non-responsiveness to aspirin treatment and a high-risk of atherothrombotic complications using two interpretable and independent methods (aggregation and PFA 100). The second objective was to find the correlation between both assays and to evaluate the results in groups at risk for various cerebrovascular diseases. Material and methods: Laboratory tests of aspirin resistance were performed in 79 patients with clinical non-responsiveness to aspirin treatment suffering from neurovascular diseases. Patients were divided into the two groups: expected low risk for aspirin resistance due to the first manifestation of a neurovascular disease $(n=34)$ and expected high risk due to the second clinical manifestation of a neurovascular disease $(n=45)$. Results: The prevalence of aspirin resistance in both groups combined as determined by the PFA-100 and CPG techniques were 50.6\% and 17.7\%, respectively. No correlation was found between the two techniques. Conclusions: No significant prevalence of aspirin resistance was demonstrated by either method despite the heterogeneous pathophysiological mechanisms. However, we are presently unable to provide an accurate opinion on the value of laboratory test result or routine monitoring in clinical neurology.

Key words: Aspirin resistance; Diagnostics; Prevalence; Neurovascular disease; Antiplatelet therapy

\section{Introduction}

Acetylsalicylic acid (ASA), commonly known as aspirin, is an effective antiplatelet drug for which a beneficial effect on the atherothrombotic complications of neurovascular diseases has been clearly demonstrated. Its anti-aggregatory effect is due to its ability to irreversibly inactivate the enzyme cyclooxygenase and subsequently inhibit the production of thromboxane A2, a potent platelet activator (1). Very ample and convincing evidence is available on its efficacy and beneficial effects, especially in the secondary prevention of arterial occlusion. According to published meta-analyses, aspirin leads to a significant reduction in the occurrences of myocardial infarctions (MI), cerebrovascular accidents (CVA) and deaths in patients with symptomatic cardiovascular diseases (2-4). Despite its effectiveness in preventing vascular events, aspirin is not able to prevent thrombosis in all patients. In 2002, an analysis of 287 studies was published that compared the results from 135,000 patients undergoing antiplatelet therapy to controls. The main endpoints were the occurrence of severe vascular occlusions, non-fatal MI, non-fatal strokes or death due to vascular disease (4). The results of this analysis suggest that antiplatelet therapy in high-risk patients is suitable and reduces the occurrence of severe vascular events, including MI, non-fatal stroke, transient ischemic attacks (TIA), unstable angina pectoris, and obliterating atherosclerosis of the arteries in the lower extremities. In addition, it reduces the risks of embolism during atrial fibrillation and vascular occlusion in other high-risk patients.

Aspirin resistance is defined as the condition in which treatment with ASA fails and cannot prevent additional thrombotic complications despite usage in an appropriate manner (it is called "clinical resistance" by some authors) (5). Aspirin resistance also refers to several different phenomena, such as the inability of aspirin to produce the prolongation of bleeding time or the reduction of platelet function in one or more platelet aggregation tests conducted in vitro ("laboratory resistance") $(6,7)$. However, "laboratory resistance" reflects a variable level of individual sensitivity to the effect of ASA (5). It is difficult to confirm ASA resistance because it is related to the clinical effects of ASA rather than to verification by laboratory tests. Direct comparison of different laboratory methods to detect aspirin resistance usually showed very weak or no correlation. One of the methods used to prove ASA resistance is the measurement of platelet aggregation with optical aggregometry after propyl gallate (CPG) induction, or the semi-automatic measurement of platelet activity (PFA 100). This technique is simple and fast, however its disadvantages include unclear sensitivity 
Tab. 1: Demographic characteristics and risk factors in the evaluated groups.

\begin{tabular}{|l|c|c|c|c|c|}
\hline Total number of men/women & Age; mean \pm SD & Arterial hypertension & Diabetes mellitus & Dyslipidemia & Smokers \\
\hline 79 & $71.9 \pm 9.1$ & $78 \%$ & $49 \%$ & $54 \%$ & $36 \%$ \\
\hline 48 men & $69.8 \pm 8.9$ & $52 \%$ & $31 \%$ & $35 \%$ & $26 \%$ \\
\hline 31 women & $74.8 \pm 9.7$ & $26 \%$ & $17 \%$ & $19 \%$ & $10 \%$ \\
\hline
\end{tabular}

and poor correlation with clinical findings. The measurement of thromboxane production using a urine thromboxane metabolite assay demonstrates a better correlation with clinical findings but possesses unclear specificity and sensitivity and, therefore, decreased reproducibility $(8,9)$.

The objective of this study was to determine the prevalence of ASA resistance in neurovascular patients with clinical non-responsiveness to aspirin treatment using two interpretable and independent methods (aggregation after the use of CPG and examination of primary hemostasis on PFA-100). The second objective was to assess the correlation between both assays and evaluate the results in various groups with the risk of cerebrovascular diseases.

\section{Material and Methods}

\section{Patients}

Laboratory tests of aspirin resistance were performed in 79 patients ( 48 men and 31 women) suffering from neurovascular diseases with clinical non-responsiveness to aspirin treatment who were hospitalized at the Department of Neurology, University Hospital in Hradec Králové. The neurovascular diseases diagnosed include first CVA attack, TIA, symptomatic stenosis or restenosis of the extracranial carotid arteries after surgical or endovascular treatment and recurrent CVA in patients treated with ASA at a dose of $100 \mathrm{mg}$ daily for at least three months. The demographic characteristics of the patients and some basic vascular risk factors (arterial hypertension, diabetes mellitus, dyslipidaemia and smoking) are shown in Table 1. Patients undergoing dual antiplatelet therapy and suffering from cardioembolic CVA were excluded. Only patients with normal ranges of platelet counts were included. Patients were divided into two groups, which are described along with their frequency rates in Table 2. Group A (expected low risk = the first clinical manifestation) consisted of patients diagnosed with CVA or TIA as the first manifestation of neurovascular disease. Group B (expected high risk = the second clinical manifestation) consisted of patients diagnosed with recurrent CVA or symptomatic stenosis or restenosis of the extracranial carotid arteries after surgical or endovascular treatment as the second clinical manifestation of a neurovascular disease.

\section{Blood sampling}

Atraumatic blood sampling was performed using a closed collection system (BD Vacutainer, Franklin Lakes,
Tab. 2: Division of the evaluated population according to diagnostic groups.

\begin{tabular}{|l|l|c|}
\hline Group & Diagnosis & Count \\
\hline A & CVA, TIA & 34 \\
\hline B & $\begin{array}{l}\text { recurrence of CVA, carotid stenosis } \\
\text { or restenosis }\end{array}$ & 45 \\
\hline
\end{tabular}

Legend: CVA - cerebrovascular accidents, TIA - transient ischemic attack.

NJ, USA) with a minimal strangulation of the arm and with a needle size of $21 \mathrm{G}$. During the collection, the first collected test-tube was discarded due to a possible platelet activation. Blood sample for the aggregation examination was collected into two tubes with $3.2 \%$ sodium citrate ( 1 portion of anticoagulant and 9 portions of blood) and for PFA-100 examination into one tube with $3.2 \%$ buffered sodium citrate ( 1 portion of anticoagulant and 9 portions of blood). Immediately after the collection, the tubes were carefully transported to the laboratory in a temperature of $15-25^{\circ} \mathrm{C}$ and blood samples were examined within 2 hours after collection.

\section{Laboratory tests}

Platelet aggregability following CPG activation and the determination of haemostatic functions using the PFA-100 (Platelet Function Analyzer) device were used as laboratory methods for the determination of aspirin resistance.

\section{Assessment of primary hemostasis using the PFA-100 device}

A Platelet Function Analyzer (PFA-100) was used to simulate primary hemostasis after vascular wall injury. Blood was collected into $3.2 \%$ buffered sodium citrate and examined within 2 hours after the collection (stability of blood sample is 4 hours after the collection for this test). We used the PFA-100 device to measure closure time $(C T)$, which is defined as the time required for blood to pass the capillary tube and is an indicator of platelet function. A collagen-coated membrane and epinephrine-coated C/Epi and $\mathrm{C} / \mathrm{ADP}$ cartridges were used for platelet adhesion. Primary hemostasis parameters (platelet adhesion and aggregation) were assessed after passing the aperture $(147 \mu \mathrm{m})$ and collagen coated membrane with the addition of ADP $(50 \mu \mathrm{g})$ and adrenaline $(10 \mu \mathrm{g})$. 


\section{Propyl gallate-induced platelet aggregation}

Propyl gallate-induced platelet aggregation employs a cationic substance (Cationic Propyl Gallate-CPG) to induce an aggregation response. Cationic Propyl Gallate (CPG) was purchased from Analytical Control Systems, Inc. The measurement was performed on an optical aggregometer using platelet-rich (PRP) and platelet poor (PPP) plasma. Blood samples were centrifuged for 15 minutes at $110 \mathrm{~g}$ to obtain a PRP. Platelet-rich plasma was separated and the tubes were again centrifuged for 15 minutes at $2000 \mathrm{~g}$ to obtain PPP (platelet count in PPP was $\leq 5 \times 10^{9} / \mathrm{L}$ ). The measuring range of the aggregometer APACT 4004 (LaBiTec Labor BioMedical Technologies $\mathrm{GmbH}$, Ahrensburg, Germany) is 30-600 PLT $\times 10^{9} / \mathrm{L}$. In all examined patients, platelet count in PRP was in a range of $146-605 \times 10^{9} / \mathrm{L}$. Thus, correction of platelet count in PRP using an autologous PPP was not necessary. We measured the height of maximum amplitude (ma), slope of the aggregation curve (ms slope) and time needed to reach 50\% ma (T50). Low platelet counts can significantly influence the test results; therefore, only patients with normal ranges $\left(150-400\right.$ PLT $\left.\times 10^{9} / \mathrm{L}\right)$ were included.

\section{Statistical analysis}

All statistical tests were performed using $R$ version 2.10.1, Copyright (C) 2009 The R Foundation for Statistical Computing with a level of significance equal to 0.05 .

We focused on the following two topics: 1) the relationship between the CPG and PFA-100 tests and 2) the relationship between the proportions of results in the $A$ and $\mathrm{B}$ groups of patients. Regardless of the test considered (CPG or PFA-100), the only possible results are R (resistance) or $\mathrm{N}$ (nonresistance). Thus, the result of the test can be considered as a random variable possessing a binomial distribution. This assumption was used for exploring both of the above-mentioned topics. The first topic was explored by the Pearson's Chi-squared test with Yates' continuity correction. The second topic was explored by the test for equality of proportions.

\section{Results}

The prevalence of a result of " $\mathrm{R}$ " in Groups A and B, as determined by the PFA-100 and $\mathrm{CPG}$ techniques, were $50.6 \%$ and $17.7 \%$, respectively.

Table 3 depicts the relationship between the results of the CPG and PFA-100 tests as determined by considering the whole cohort of 79 patients. It can be seen that for seven patients, although the CPG test result was R, the PFA-100 test result was N. Conversely, there were 33 patients with a result of $R$ in the PFA-100 test that had a result of $N$ in the $\mathrm{CPG}$ test. If the results of these two tests are dependent random variables (meaning that the tests are commutable), then the counts of $\mathrm{R} \times \mathrm{N}$ or $\mathrm{N} \times \mathrm{R}$ test results should not be significant.
Tab. 3: The relationship between results of CPG and PFA.

\begin{tabular}{|l|c|c|c|}
\hline \multirow{2}{*}{ CPG } & \multicolumn{2}{|c|}{ PFA-100 } & \multirow{2}{*}{$\Sigma$} \\
\cline { 2 - 3 } & $\mathrm{N}$ & $\mathrm{R}$ & \\
\hline $\mathrm{N}$ & 32 & 33 & 65 \\
\hline $\mathrm{R}$ & 7 & 7 & 14 \\
\hline $\mathrm{\Sigma}$ & 39 & 40 & 79 \\
\hline
\end{tabular}

Legend: CPG - propyl gallate-induced platelet aggregation,

PFA-100 - platelet function analyzer, $\mathrm{R}$ - resistance, $\mathrm{N}$ - nonresistance.

$R$ version 2.10.1 provides a Pearson's Chi-squared test with the Yates' continuity correction.

There is no relationship of similarity between the results of the given two tests. As was already mentioned, the result of the blood condensability test can be considered as a random variable with a binomial distribution. In this work, the parameter $n$ represents the number of tested patients, and the parameter $p$ denotes the probability of a test result of R. Furthermore, $p_{A}$ and $p_{B}$ denote the probabilities of a test result of $\mathrm{R}$ within Groups $\mathrm{A}$ and $\mathrm{B}$, respectively. Table 4 depicts the results of both blood tests.

Tab. 4: The results of both blood tests.

\begin{tabular}{|l|c|r|r|r|c|}
\hline \multirow{2}{*}{ Group } & \multicolumn{2}{|c|}{ CPG } & \multicolumn{2}{c|}{ PFA-100 } & \multirow{2}{*}{$\Sigma$} \\
\cline { 2 - 5 } & $\mathrm{N}$ & $\mathrm{R}$ & $\mathrm{N}$ & $\mathrm{R}$ & \\
\hline $\mathrm{A}$ & 27 & 7 & 12 & 22 & 34 \\
\hline $\mathrm{B}$ & 38 & 7 & 27 & 18 & 45 \\
\hline$\Sigma$ & 65 & 14 & 39 & 40 & 79 \\
\hline
\end{tabular}

Legend: $C P G$ - propyl gallate-induced platelet aggregation,

PFA-100 - platelet function analyzer, $\mathrm{R}$ - resistance, $\mathrm{N}$ - nonresistance.

Table 5 depicts the values of $\pi_{A}$ and $\pi_{B}$, which denote the estimates of $p_{A}$ and $p_{B}$, respectively.

For both blood tests, the result of statistical testing was that the hypothesis could not be rejected ( $\mathrm{p}$-values equal to 0.7776 and 0.05148 , respectively). No correlation was found between the two techniques, and neither group demonstrated a statistically higher probability of resistance. This demonstrates that there is no significant prevalence that either of the groups is more resistant.

Tab. 5: The values of $\pi_{A}$ and $\pi_{B}$, which denote the estimates of $p_{A}$ and $p_{B}$.

\begin{tabular}{|l|c|c|}
\hline Test & $\boldsymbol{\pi}_{\boldsymbol{A}}$ & $\boldsymbol{\pi}_{\boldsymbol{B}}$ \\
\hline CPG & 0.21 & 0.16 \\
\hline PFA-100 & 0.65 & 0.40 \\
\hline
\end{tabular}

Legend: CPG - propyl gallate-induced platelet aggregation, PFA-100 - platelet function analyzer. 


\section{Discussion}

Data on the prevalence of aspirin resistance in the literature are highly variable in different groups of patients, ranging from 5.2 to $69 \%$ in patients with stable ischemic heart disease, from 22.5 to $83.3 \%$ in patients with acute coronary syndrome and from 20 to $74 \%$ in patients early after undergoing aortocoronary bypass graft surgery (10-14). Aspirin resistance was observed in 5 to $60 \%$ of patients with a cerebrovascular disorder after CVA, and in 9 to $65 \%$ of patients with peripheral arterial disease of the lower extremities (15).

The causes of aspirin resistance are not completely understood and their determination is subject to discussion. Clinical factors, such as low dosage, noncompliance, increase of platelet aggregation induced by external factors (smoking, stress), arteritis and cellular factors (regenerated, non-inhibited cyclooxygenase 1 in nucleated cells, increased sensitivity of platelets to collagen and ADP, elevation of noradrenaline) contribute to the development of aspirin resistance. Genetic polymorphisms (cyclooxygenase 1 and 2, thromboxane $\mathrm{A}_{2}$ synthetase, GPIa/IIa, Ib/V/IX, IIb/ IIIa receptor, collagen receptor) have also been discussed as possible causes of aspirin resistance $(9,16,17)$. Aspirin resistance is difficult to define, and its laboratory determination is difficult. The prevalence reported in the literature varies according to the methods and techniques used (see Table 6).

It was demonstrated in several studies that patients with ASA resistance have a higher frequency of fatal and non-fatal coronary episodes than the responders. Aspirin resistance is considered to be a new risk factor of arterial thrombosis $(12,18)$. Most studies demonstrated that the impaired sensitivity to ASA (detected by multiple laboratory methods) had a negative clinical impact on both spontaneous episodes (death of cardiovascular causes, CVA, peripheral arterial disease) and procedure complications (reocclusion, thrombosis) $(7,19)$.

Our results, as assessed by the two independent methods of PFA-100 and CPG, correspond with the prevalence of aspirin resistance as reported in a wide range in the world literature $(50.6 \%$ and $17.7 \%)(13,20)$. No significant correlation between the two tests was demonstrated. The statistical evaluation of two different high-risk groups of cerebrovascular diseases failed to confirm a higher probability of resistance in either group. The first group of patients (Group A) included patients with CVA or TIA as their first clinical manifestation of neurovascular disease and had a lower expected risk of aspirin resistance, CVA and TIA. The second group (Group B) included patients who had already suffered from a secondary cerebrovascular disease (recurrent CVA or symptomatic stenosis or restenosis of the extracranial carotid arteries after surgical or endovascular treatment) and had a higher expected risk of aspirin resistance. There is no significant difference in prevalence when comparing one group to the other as determined by either laboratory test, despite the heterogeneous etiopathophysiological mechanisms and different degree of the involvement of platelets in the formation of thrombotic occlusion in these two groups, influencing the limits of the ASA efficacy.

Despite a wide range of tests designed to monitor the efficacy of antiplatelet drugs, it remains to be determined how much clinical resistance correlates with laboratory results. It can be concluded that resistance to antiplatelet therapy is a pathophysiological phenomenon with possible clinical relevance. The mechanisms of aspirin and clopidogrel resistance are influenced by many factors, including genetic ones, and the variability of response to antiplatelet therapy is multifactorial (21-23). Although much information has been obtained, ASA resistance remains an open issue with many questions. The crucial question is whether ASA resistance has clinical relevance or is simply a laboratory phenomenon. We do not know the actual compliance rate of patients undergoing ASA therapy (the monitoring of ASA metabolites in urine for the potential determination of compliance is a topic for future research) nor do we know the prevalence of ASA resistance in patients with coronary syndromes or neurovascular diseases. We would like to know whether the resistance

Tab. 6: Selected studies and laboratory tests to detect aspirin resistance.

\begin{tabular}{|l|c|l|l|c|}
\hline Study & $\begin{array}{c}\text { ASA dose } \\
\text { in mg }\end{array}$ & $\begin{array}{l}\text { Method for determination } \\
\text { of ASA resistance }\end{array}$ & $\begin{array}{l}\text { Criteria of ASA } \\
\text { resistance }\end{array}$ & $\begin{array}{c}\text { Prevalence of } \\
\text { ASA resistance }\end{array}$ \\
\hline $\begin{array}{l}\text { Patients after aortocoronary } \\
\text { bypass graft (Buchanan et al.) }\end{array}$ & 325 & Bleeding time & $\begin{array}{l}\text { No prolongation } \\
\text { of bleeding time }\end{array}$ & $43.0 \%$ \\
\hline $\begin{array}{l}\text { Patients with stable ischemic } \\
\text { heart disease (Gumm et al.) }\end{array}$ & 325 & $\begin{array}{l}\text { PFA 100 + ADP and collagen } \\
\text { aggregation }\end{array}$ & $\begin{array}{l}\text { Normal time after col- } \\
\text { lagen and adrenaline }\end{array}$ & $9.5 \%$ \\
\hline $\begin{array}{l}\text { Patients after sudden } \\
\text { crebrovascular accident } \\
\text { (Helgason et al.) }\end{array}$ & 325 & $\begin{array}{l}\text { Aggregometry, ADP, EPI, } \\
\text { Col, ARA }\end{array}$ & & $25.0 \%$ \\
\hline $\begin{array}{l}\text { Patients with stable angina } \\
\text { (Macchi et al.) }\end{array}$ & 160 & PFA 100 & $\begin{array}{l}\text { Closure time } \\
<185 \mathrm{~s}\end{array}$ & $29.2 \%$ \\
\hline $\begin{array}{l}\text { Patients after sudden cerebro- } \\
\text { vascular accident (Eikelboom } \\
\text { et al.) }\end{array}$ & 325 & $\begin{array}{l}\text { Urine levels of 11-dehydro- } \\
\text { thromboxane B }\end{array}$ & $\begin{array}{l}\text { Increased compared } \\
\text { to the control group }\end{array}$ & $\begin{array}{c}\text { Prevalence not } \\
\text { determined }\end{array}$ \\
\hline
\end{tabular}

Legend: ASA - acetylsalicylic acid, PFA-100 - platelet function analyzer. 
to antiplatelet therapy is a permanent or transient phenomenon (related to the variability of laboratory results). Another important question pertains to intraindividual variability (24). Given the aforementioned importance of dual antiplatelet therapy in patients after coronary intervention, the potential clinical relevance of clopidogrel resistance should also be mentioned; however, there is a lack of clinical data to support this (25). No standardized technique for the detection of antiplatelet therapy efficacy has been determined to date.

Methods for the determination of antiplatelet therapy efficacy currently have low specificities and sensitivities $(7,15)$. The issue of resistance to antiplatelet therapy has raised many questions in the area of neurovascular diseases (26-28).

The heterogeneity and relatively small sizes of the evaluated groups represent the main limitations of the present study. Also, we were not able to exclude a possible noncompliance, representing of course a major problem when comparing different laboratory methods used for the assessment of the medicine efficacy without simultaneous proof of this medicine or its metabolite in the organism. However, the determination of aspirin resistance by two independent methods and the assessment of its correlation represent positive aspects of this study.

\section{Conclusions}

Many studies need to be done to answer basic questions on its clinical utility and cost-effectiveness, before antiplatelet monitoring can be recommended in the clinical practice. However, presently, we are unable to provide an accurate opinion on the clinical relevance of laboratory resistance or routine monitoring of resistance to platelet therapy in clinical neurology.

\section{Acknowledgements and contributions}

Supported by the grant MH CZ-DRO (UHHK, 00179906) and partially by the grant projects number FNHK 00179906 and PRVOUK: P37/08.

\section{References}

1. Michelson AD, Cattaneo M, Eikelboom JW, et al.; Platelet Physiology Subcommittee of the Scientific and Standardization Committee of the International Society on Thrombosis and Haemostasis; Working Group on Aspirin Resistance. Aspirin resistance: position paper of the Working Group on Aspirin Resistance. J Thromb Haemost 2005; 3: 1309-11.
2. Antithrombotic Trialists' (ATT) Collaboration. Aspirin in the primary and secondary prevention of vascular disease: collaborative meta-analysis of individual participant data from randomised trials. Lancet 2009; 373: 1849-60.

3. Berger JS, Krantz MJ, Kittelson JM, et al. Aspirin for the prevention of cardiovascular events in patients with peripheral artery disease: a meta-analysis of randomized trials. JAMA 2009; 301: 1909-19.

4. Antithrombotic Trialists' Collaboration. Collaborative meta-analysis of randomised trials of antiplatelet therapy for prevention of death, myocardial infarction, and stroke by prolonged antiplatelet therapy in high-risk patients. BMJ 2002; 324: 71-86.

5. Cattaneo M. Resistance to antiplatelet drugs: molecular mechanisms and laboratory detection. J Thromb Haemost 2007; 5(Suppl 1): 230-7.

6. Cheng X, Chen WH, Simon DI. Aspirin resistance or variable response or both? Am J Cardiol 2006; 98: 11-7.

7. Patrono C, Baigent C, Hirsh J, Roth G; American College of Chest Physicians. Antiplatelet drugs: American College of Chest Physicians Evidence-Based Clinical Practice Guidelines (8th Edition). Chest 2008; 133(6 Suppl): 199S-233S.

8. Mason PJ, Freedman JE, Jacobs AK. Aspirin resistance: current concepts. Rev Cardiovasc Med 2004; 3: 156-63.

9. McKee SA, Sane DC, Deliargyris EN. Aspirin resistance in cardiovascular disease: a review of prevalence, mechanisms and clinical significance. Thromb Haemost 2002; 88: 711-5.

10. Buchanan MR, Bister SJ. Individual variation in the effects of ASA on platelet function: implications for the use of ASA clinically. Can J Cardiol 1995; 11:221-7.

11. Eikelboom JW, Hirsh J, Weitz JI, et al. Aspirin-resistant thromboxane biosynthesis and the risk of myocardial infarction, stroke, or cardiovascular death in patients at high risk for cardiovascular events. Circulation 2002; 105: 1650-5.

12. Gum PA, Kottke-Marchant K, Poggio ED, et al. Profile and prevalence of aspirin resistance in patients with cardiovascular disease. Am J Cardiol 2001; 88: 230-4.

13. Helgason CM, Bolin KM, Hoff JA, et al. Development of aspirin resistance in persons with previous ischemic stroke. Stroke 1994; 25: 2331-6.

14. Macchi L, Christiaens L, Brabant S, et al. Resistance to aspirin in vitro is associated with increased platelet sensitivity to adenosine diphosphate. Thromb Res 2002; 107: 45-9.

15. Zimmermann N, Hohlfeld T. Clinical implications of aspirin resistance. Thromb Haemost 2008; 100: 379-90.

16. Hankey GJ, Eikelboom JW. Aspirin resistence. Lancet 2006; 367: 606-7.

17. Wang TH, Bhatt DL, Topol EJ. Aspirin and clopidogrel resistance: an emerging clinical entity. Eur Heart J 2006; 27: 647-54.

18. Gum PA, Kottke-Marchant K, Welsh PA, et al. A prospective, blinded determination of the natural history of aspirin resistance among stable patients with cardiovascular disease. JACC 2003; 41: 961-5.

19. Krasopoulos G, Brister SJ, Beattie WS, et al. Aspirin "resistance" and risk of cardiovascular morbidity: systematic review and meta-analysis. BMJ 2008; 336: 195-8.

20. Sztriha LK, Sas K, Vecsei L. Aspirin resistance in stroke: 2004. J Neurol Sci 2005; 229/230: 163-9.

21. Lev EI, Patel RT, Maresh KJ, et al. Aspirin and clopidogrel drug response in patients undergoing percutaneous coronary intervention: the role of dual drug resistance J Am Coll Cardiol 2006; 47: 27-33.

22. Fifi JT, Brockington C, Narang J, et al. Clopidogrel resistance is associated with thromboembolic complications in patients undergoing neurovascular stenting. Am J Neuroradiol 2013; 34: 716-20.

23. Mijajlovic MD, Shulga O, Bloch S, Covickovic-Sternic N, Aleksic V, Bornstein NM. Clinical consequences of aspirin and clopidogrel resistance: an overview. Acta Neurol Scand 2013; 128: 213-9.

24. Addad F, Hassine M, Ben Farhat M, et al. Intra-individual variability of the platelet anti-aggregation effect of aspirin in coronary patients. Arch Mal Coeur Vaiss 2005; 98: 979-83.

25. Oqueli E, Hiscock M, Dick R. Clopidogrel resistance. Heart Lung Circ 2007; 16 Suppl 3: S17-28.

26. Greer DM. Aspirin and antiplatelet agent resistance: implications for prevention of secondary stroke. CNS Drugs 2010; 24: 1027-40.

27. Jeon SB, Song HS, Kim BJ, et al. Biochemical aspirin resistance and recurrent lesions in patients with acute ischemic stroke. Eur Neurol 2010; 64: 51-7.

28. Cattaneo M. High on-treatment platelet reactivity - definition and measurement Thromb Haemost 2013; 109: 792-8.

\section{Corresponding author:}

Assoc. Prof. Martin Vališ, MD, PhD, Department of Neurology, University Hospital in Hradec Králové, Sokolská 581, CZ-500 05 Hradec Králové, Czech Republic; e-mail: Valismar@seznam.cz 